\title{
Cardiac involvement in lymphoma mimicking hypertrophic cardiomyopathy
}

\author{
Petr Kuchynka ${ }^{1}$, Tomas Palecek ${ }^{1}$, Lukas Lambert ${ }^{2}$, Martin Masek $^{2}$, Valerie Knotkova ${ }^{3}$ \\ ${ }^{1} 2^{\text {nd }}$ Department of Medicine - Department of Cardiovascular Medicine, $1^{\text {st }}$ Faculty of Medicine, Charles University and General \\ University Hospital in Prague, Prague, Czech Republic \\ 2Department of Radiology, $1^{\text {st }}$ Faculty of Medicine, Charles University and General University Hospital in Prague, Prague, Czech Republic \\ ${ }^{3}$ Institute of Nuclear Medicine, $1^{\text {st }}$ Faculty of Medicine, Charles University and General University Hospital in Prague, Prague, Czech Republic
}

Cardiac involvement in lymphoma represents a rare type of myocardial pathology. The right side of the heart is most frequently involved, especially the right atrium [1]. Epicardial and pericardial infiltration with pericardial effusion are regarded as typical [2]. We describe an unusual case of biventricular myocardial thickening caused by infiltration due to non-Hodgkin lymphoma and mimicking the phenotype of hypertrophic cardiomyopathy (HCM). A 38-year-old man with a month-long history of abdominal lymphadenopathy was referred to our department for evaluation of pericardial effusion and a suspicion of HCM. Transthoracic echocardiography showed non-dilated left and right ventricles with significantly reduced systolic function, impaired left ventricular relaxation, and diffuse thickening of ventricular walls (interventricular septum $24 \mathrm{~mm}$, right ventricle free wall $18 \mathrm{~mm}$ ). Moreover, dilatation of both atria and thickening of the interatrial septum with large pericardial effusion was present (Fig. 1, Suppl. Video 1 - see journal website). In order to better characterise cardiac involvement, cardiac magnetic resonance (CMR) was performed (Suppl. Video 2 - see journal website). CMR depicted not only previously described heart pathologies but also showed diffuse inhomogeneous late gadolinium enhancement in the myocardium of both ventricles and interatrial septum (IAS), highly suspicious of myocardial involvement associated with probable lymphoproliferative disease (Fig. 2). To clarify the cause of lymphadenopathy, positron emission tomography-computed tomography was performed, and generalised lymphadenopathy typical of for lymphoproliferative haematological disorder was found. Regarding the cardiac involvement, high inhomogeneous uptake

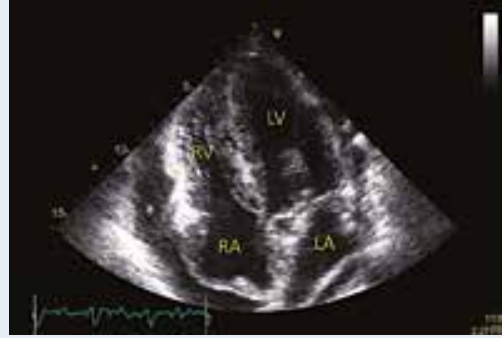

Figure 1. Transthoracic echocardiography demonstrating increased wall thickness of both ventricles and interatrial septum in the apical four-chamber view. Pericardial effusion is marked by an asterisk; LA — left atrium; LV — left ventricle; $\mathrm{RA}$ — right atrium; RV — right ventricle

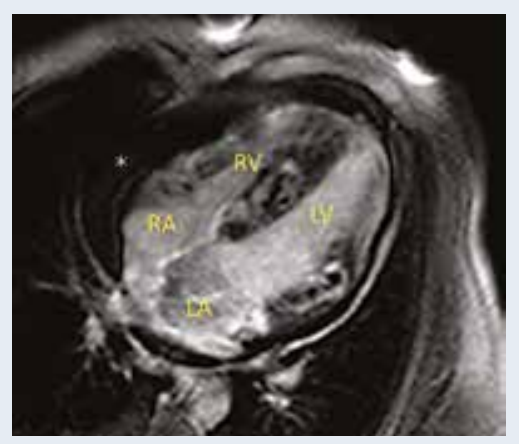

Figure 2. Cardiac magnetic resonance depicting diffuse inhomogeneous late gadolinium enhancement in both ventricles and interatrial septum. Pericardial effusion is marked by an asterisk; abbreviations - see Figure 1



Figure 3. Positron emission tomography-computed tomography demonstrating high inhomogeneous uptake of 18-fluoro-deoxyglucose in the myocardium of both ventricles and interatrial septum. Pericardial effusion is marked by an asterisk; abbreviations - see Figure 1

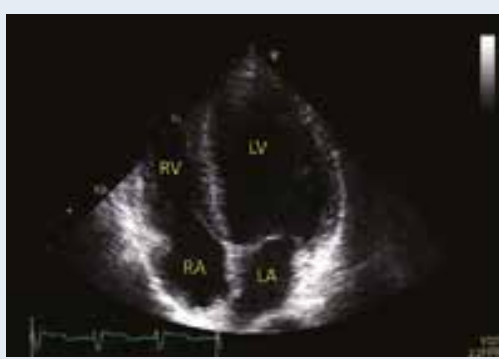

Figure 4. Transthoracic echocardiography showing normalisation of ventricular wall thickness and systolic function and disappearance of pericardial effusion in apical four-chamber view; abbreviations — see Figure 1 of 18-fluoro-deoxyglucose in the myocardium of both ventricles and IAS was detected (Fig. 3). The patient underwent surgical extirpation of the enlarged lymph node from the left groin, and non-Hodgkin lymphoma was diagnosed. After four cycles of chemotherapy including corticosteroids complete remission was achieved. This treatment led to normalisation of ventricular wall thickness and systolic function of both ventricles as well as disappearance of pericardial effusion (Fig. 4, Suppl. Video 3 - see journal website), which definitively confirmed our suspicion of cardiac involvement associated with lymphoma infiltration. In differential diagnosis of biventricular myocardial thickening accompanied by pericardial effusion many entities might be considered, including a broad spectrum of sarcomeric HCM phenocopies and infiltrative diseases such as cardiac amyloidosis. The latter can result in a significant increase in the thickness of the left and right ventricular myocardium, IAS, and pericardial effusion, as was shown in our case. However, the presence of only mild diastolic dysfunction in an advanced form of cardiac amyloidosis would be highly unlikely.

\section{References}

1. Voigt P, Wienbeck S, Weber MA, et al. Cardiac hematological malignancies: typical growth patterns, imaging features, and clinical outcome. Angiology. 2018; 69(2): 170-176, doi: 10.1177/0003319717713581, indexed in Pubmed: 28602141.

2. Jeudy J, Burke A, Frazier A. Cardiac Lymphoma. Radiol Clin North Am. 2016; 54(4): 689-710, doi: 10.1016/.r.rl.2016.03.006.

\section{Address for correspondence:}

Tomas Palecek, MD, PhD, $2^{\text {nd }}$ Department of Medicine - Department of Cardiovascular Medicine, General University Hospital in Prague, $1^{\text {st }}$ Faculty of Medicine, Charles University in Prague, U Nemocnice 2, 12808 Prague 2, Czech Republic, tel: + 4202 24962634, fax: + 4202 24912154, e-mail: tpalec@lf1.cuni.cz

Conflict of interest: none declared

Kardiologia Polska Copyright (c) Polish Cardiac Society 2018 